\title{
Les larmes de Rome. Le pouvoir de pleurer dans
} l'Antiquité

Paris, Anamosa, 2017, pp. 256, ISBN 979-10-95772-30-9, € 21,00

\section{Alfredo Casamento}

\section{OpenEdition}

\section{Journals}

Edizione digitale

URL: http://journals.openedition.org/mythos/390

DOI: $10.4000 /$ mythos.390

ISSN: 2037-7746

\section{Editore}

Salvatore Sciascia Editore

\section{Edizione cartacea}

Data di pubblicazione: 1 dicembre 2018

Paginazione: 171-174

ISBN: 978-88-8241-501-3

ISSN: $1972-2516$

\section{Notizia bibliografica digitale}

Alfredo Casamento, «Les larmes de Rome. Le pouvoir de pleurer dans l'Antiquité », Mythos [Online],

12 | 2018, online dal 24 septembre 2019, consultato il 25 septembre 2020. URL : http:// journals.openedition.org/mythos/390 ; DOI : https://doi.org/10.4000/mythos.390 


\title{
Sarah Rey
}

\author{
Les larmes de Rome. Le pouvoir de pleurer dans l'Antiquité
}

Paris, Anamosa, 2017, pp. 256, ISBN 979-10-95772-30-9, € 21,00.

Alfredo Casamento - Università degli Studi di Palermo - alfredo.casamento@unipa.it

Barack Obama che piange dopo aver presentato una proposta di legge volta a limitare il ricorso alle armi e di nuovo, pochi mesi dopo, lasciando la Casa Bianca a conclusione del secondo mandato presidenziale è la stimolante immagine che apre il volume di Sarah Rey. II tono provocatorio di questa rappresentazione inquadra molto bene, e fin dalle prime battute, questo piccolo ma prezioso volume che ha l'ambizione di mettere insieme un tema di ricerca assai importante per il mondo antico con una pregevole operazione di divulgazione presso un pubblico ampio, non necessariamente di specialisti.

Diciamo subito che la riflessione sulle lacrime nella cultura antica ha ricevuto nell'ultimo ventennio un'attenzione solo all'apparenza sorprendente, in quanto essa si iscrive in un più generale aggiornamento delle ricerche sulle emozioni nella tradizione greca e latina. In quest'ottica, gli studi sulle lacrime si sono distinti perché su di esse si sono ritrovate in maniera convergente prospettive di tipo linguistico, semiotico, antropologico, storico-culturale. Ultimo in ordine di tempo tra gli studi di carattere collettaneo che affronta in maniera monotematica e da molti punti di vista l'argomento è il volume del 2009 di T. Fögen (Tears in the Graeco-Roman World, Berlin-New York 2009). Di questo sforzo di rendere conto delle molteplici, variegate declinazioni del motivo I'A. dà conto nella premessa, nella quale con brillantezza d'immagini riunisce alcuni dei temi che avranno debita trattazione nel corso del volume. Dopo aver delimitato il campo d'indagine allo specifico del mondo latino ("à chaque culture ses émotions"), la studiosa giustifica la scelta, ricordando la ricchezza che contraddi- stingue il campo semantico, o forse sarebbe meglio dire i campi semantici, del pianto nella lingua latina, secondo l'idea che a questo articolato ventaglio di termini corrisponde una ricchezza di impieghi talmente ampia da risultare arduo seguirne tutti gli sviluppi.

Sei brevi ed intensi capitoli scandiscono lo sguardo accurato sul pianto nel mondo romano, secondo una prospettiva che appare opportuno documentare con maggiore precisione al fine di rendere la dovuta attenzione a questo pregevole lavoro.

Nel corso del primo capitolo, Pleurer les morts (pp. 21-52), si affronta il tema tradizionale del piangere i morti cogliendo immediatamente i nessi che legano aspetti squisitamente religiosi e devozionali con quelli che rimandano all'antropologia, alla storia delle idee, al diritto. Così, dopo un brillante inizio in cu ricorda che "dans l'expression de la douleur, le droit et la politique trouvent leur mot à dire" ( $p$. 21), l'A. menziona l'interdizione stabilita dalle Leggi delle Dodici Tavole, che vietavano alle donne un particolare tipo di lamento, il lessus, considerato eccessivo. Attenzione specifica è poi dedicata alle iscrizioni funebri, nelle quali le lacrime sono riservate alla cerchia ristretta dei familiari; interessante lo scambio tra sposi testimoniato da CIL V, 2411, in cui il defunto sollecita chi è rimasto in vita a interrompere i lamenti in considerazione dell'ordinarietà del percorso cui tutti sono destinati (quid quereris fatis mortis carissime coniunx /cum sit communis omnibus una via/desine sollicitum pectus lacerare dolore; /temporis hospitium non solet esse diu). D'altra parte, il pianto per un defunto imponeva precise ‘regole d'ingaggio', come il divieto di versar lacrime per un neona- 
to morto entro un certo tempo dalla nascita, regola che le fonti trasmettono in modo contraddittorio e che evidentemente si collega con lo statuto attribuito al bambino ancora in fasce. Altri casi celebri di interdizione del pianto sono quelli connessi a gravi momenti di crisi dello stato repubblicano, come viene testimoniato a proposito della battaglia di Canne (Val. Max. $1,1,15)$, allorquando alle donne fu vietato di piangere e portare il lutto per i soldati caduti per più di un mese. II lutto

In qualche modo connessa al primo capitolo è poi la materia sviluppata nel secondo, Et dans les temples, l'ivoire pleure (pp. 53-76), nel corso del quale l'A. mette insieme alcuni esempi relativi ai rapporti tra pianto e religione. Esemplare della considerazione 'politica' attribuibile al pianto è poi la supplicatio, la pratica rituale connessa a situazioni di crisi della res publica che meritano uno sforzo collettivo al fine di stornare minacce e pericoli incombenti sulla comunità come testimonia Livio $(26,9,6-8)$ a proposito della seconda guerra punica: "la supplicatio, cette déploration généralisée, entre dans un arsenal de mesures religieuses destinées à sauver Rome du péril cathaginois" (p. 58). Rientra a pieno titolo nella sfera religiosa l'accoglienza destinata all'introduzione della Magna Mater nel 204 a.C., i cui sacerdoti, tra gli altri elementi del rito annuale con cui ne celebrano la diffusione in città, piangono in ricordo di Attis. La trattazione si chiude poi con i casi di celebri di pianti di statue, vero topos della narrazione storiografica, a proposito del quale il saggio ribadisce, con ricca testimonianza, il carattere predittivo e ominoso.

Terzo e quarto capitolo costituiscono poi la parte più ambiziosa del volume, centrando l'analisi sulle implicazioni socio-culturali del pianto. Ad aprire la trattazione sono, nel terzo capitolo, le lacrime di Cesare: quelle versate davanti a una statua di Alessandro Magno, del quale il futuro dittatore invidiava i molteplici successi ottenuti pur giovanissimo ed il pianto esibito in Egitto dinnanzi alla testa mozzata di Pompeo. All'analisi si sarebbe forse potuta aggiungere la citazione del medesimo epi- sodio offerta da Lucano nel nono libro della Pharsalia (vv. 1035-1108), perché in questa circostanza il poeta lavora sulla serietà delle lacrime di Cesare, dimostrando con pungente ironia che si trattò di un pianto a comando, certamente insincero, esibito con astuzia politica. II valore suasivo del pianto è poi letto in prospettiva storica, documentando alcuni casi celebri in cui esso viene adoperato come mezzo per implorare i potenti: così le lacrime delle Sabine, quelle di Volumnia e Veturia, rispettivamente moglie e madre di Coriolano, o ancora quelle di Lucrezia, vittima degli appetiti di Sesto Tarquinio, costituiscono esempi significativi di una sacralizzazione del pianto, come dimostra ad esempio la fondazione del culto della Fortuna muliebre, celebrato con la fondazione di un tempio a poca distanza da Roma voluto dalle donne che con le loro suppliche avevano dissuaso Coriolano dal marciare in armi contro la città. Che il pianto coincida nei fatti con una raffinata strategia politica è poi documentato attraverso alcuni esempi. Su tutti emergono le lacrime di Augusto nell'atto di ricevere, per bocca di Valerio Messala Corvino, il titolo di pater patriae nel 2 a.C. Sincere o meno che possano essere state, contribuiscono a umanizzare volto e strategie politiche del nuovo padrone di Roma che con sguardo benevolo, proprio perché piangente, auspica il mantenimento della pace (Suet. Aug. 58, 1-3).

Altro topos storiografico che prevede il pianto è quello relativo alle lacrime del comandante dinnanzi alle rovine di una città annientata dalla potenza romana: è il caso di Camillo, davanti ai resti di Veio (Plut. Cam. 5, 7-8) o ancora di Marcello, che piange non appena espugnata Siracusa (Liv. 25, 24, 11). II valore della lacrime oscilla pericolosamente come un metronomo in accordo alla persona che piange o alla contingenza che favorisce il pianto. Così, ad esempio, dei singhiozzi di Gneo Papirio Carbone, nemico di Silla e vittima delle proscrizioni, si potrà affermare che ricordano i pianti di una donna (Val. Max. 9, 13).

Completa poi questo dittico, volto ad indagare più propriamente gli aspetti socio-rela- 
zionali del pianto, il quarto capitolo (pp. 123156) che ha come centro focale l'eloquenza e il modo con cui la retorica latina teorizza ed impiega un ricorso costante alle lacrime come elemento di persuasione. L'esempio calzante è quello di Servio Sulpicio Galba, vittima, probabilmente non incolpevole, di un processo 'mediatico' del tempo, relativo a una condotta di guerra. Siamo nel 149 a.C., Galba è chiamato a difendersi del massacro di Lusitani di cui si era responsabile in Spagna. La difesa doveva essere abbastanza periclitante, ma fu proprio l'abilità di Galba di muovere a compassione giudici e pubblico con un ampio ricorso al pianto a valergli l'assoluzione. In particolare, le fonti antiche concordano unanimemente nel sottolineare che a destare il picco della commozione fu un bambino, di cui egli era tutore poiché privo del padre, un altro Sulpicio assai rimpianto in città, e che a detta dell'oratore sarebbe rimasto solo al mondo se egli fosse stato condannato. Notevoli in questa prospettiva le osservazioni dell'A. relative al rapporto che intercorre tra eloquenza pronunciata e eloquenza scritta. Proprio il passaggio alla forma scritta testimonia, infatti, della volontà dell'oratore di dar forma concreta alle manifestazioni del pianto che dunque si elevano da una dimensione squisitamente performativa fino a raggiungere un elemento cardine delle strategie retoriche. Quando poi il discorso si sposta sul versante dell'oratoria politica (p. 144 ss.), l'A. ricorda alcuni episodi della storia di Roma - il discorso di Tarquinio agli abitanti di Tarquinia perché lo aiutino a fare rientro a Roma o quello di Cesare che sta per oltrepassare in armi il Rubicone - che la ricostruzione offerta dalle fonti presentano come casi celebri di ricorso al pianto come elemento di persuasione.

II quinto capitolo (pp. 157-185) riposiziona poi lo sguardo sulle lacrime "comme objet philosophique". L'A. riflette, infatti, sulla necessità imposta dalla maggior parte delle correnti filosofiche di trattenere il pianto, considerato attitudine femminile e dunque non adatto all'uomo che deve dar prova di saldezza e coerenza morale nei travagli della vita. Le lacrime sono percepite come una manifestazione di irragionevolezza e mancanza di riflessione, il frutto di un calcolo disperato o la sottomissione dell'uomo a pulsioni basse. È questo il caso dell'aneddoto che Cicerone mette in bocca a Catone nel De senectute (par. 27), citando il celebre atleta Milone di Crotone, che guarda, ormai vecchio, dei giovani atleti all'inizio della loro carriera. Milone piange alla vista dei giovani e dei propri muscoli che gli sembrano come morti. Ma Catone non mostra esitazione alcuna: nessun detto gli sembra più spregevole di questo (quae enim vox potest esse contemptior quam Milonis Crotoniatae). La rigorosa rivendicazione della coerenza morale del saggio fa sì che di lì a poco anche Seneca puntualizzi la necessità che l'uomo si tenga lontano dal pianto: anche ad ammettere che spesso le lacrime piombano inaspettatamente nella vita, esse però non recano alcuna utilità e come tali vanno respinte (lacrimae nihil profuturae cadunt, in Ep. ad Luc. 22, 16). Su queste premesse il capitolo si chiude, infine, con una riflessione sul valore della consolazione e sui topoi compositivi della letteratura specifica che su questo tema si realizza tra Grecia e Roma.

Nel corso del sesto capitolo (pp. 187-215), lo studio si sposta infine all'età cristiana, mettendo a fuoco il modo con cui la riflessione teologica è intervenuta a modificare il senso antico del pianto ("comment l'essor de la foi chrétienne a-t-il modifié l'ordre romain des larmes?"). L'aspetto maggiormente messo a fuoco del capitolo è quello relativo alla valorizzazione del dolore e del pianto ("les larmes font partie d'une gymnastique de l'ascèse"). II pianto è il modo convenzionale per valorizzare la risposta emotiva e simpatetica ai mali del mondo, anche se un ricorso eccessivo alle lacrime come quello dell'eresia montanista sarà oggetto di vibrante contestazione.

Conclude il volume un ultimo capitolo (pp. 217-225) che trae avvio dal pianto del grande oratore Ortensio, di cui ancora più di un secolo dopo Quintiliano ricorderà le straordinarie qualità psicagogiche. Qualità che, più in generale, hanno molto a che fare con le lacrime. II 
pianto, infatti, si situa entro un preciso sistema di riferimento; esso è il frutto di una strategia e, come dimostra l'interesse della retorica sul punto, offre un'arma formidabile all'oratore che di esso dovrà servirsi mettendone a frutto le molteplici potenzialità: "les sanglots viennent au point d'achèvement d'un raisonnement, sans couper court à l'argumentation".
Corredano il volume utili indici e un pregevole apparato di immagini, l'ultima delle quali - un fotogramma tratto dal film Quo vadis in cui Nerone raccoglie in un'ampolla le sue lacrime versate in memoria dell'amico Petronio, del quale aveva pur richiesto la morte - suggella questo intenso e appassionato percorso nel pianto dei Romani. 